\title{
Drip Fertigation's Effect on Nutrient Contents of Chiili (Capsicum annuum)
}

\author{
C. Ciba $^{1^{*}}$ and M. Syamala ${ }^{2}$ \\ ${ }^{1}$ Department of Horticulture, ${ }^{2}$ Department of Plant Pathology, Agricultural College and Research \\ Institute, Madurai, Tamil Nadu Agricultural University, Coimbatore -641003, Tamil Nadu, India \\ *Corresponding author
}

\section{A B S T R A C T}

A field experiment on nutrient contents like $\mathrm{N}, \mathrm{P}, \mathrm{K}$ and micro nutrient of chiili

Keywords

Drip Fertigation,

Nutrient contents and micro nutrients, Chill

Article Info

Accepted:

24 September 2017

Available Online:

10 November 2017 (capsicum anпиит. L) cv. KKM-1 under drip fertigation was carried out at Madurai, Tamil Nadu during kharif and summer. The experiments were laid out in Randomized Block Design (RBD) with nine treatments in three replications. The study revealed that the increased nutrient content was obtained in $T_{9}\left(T_{5}+\right.$ liquid biofertilizers + Panchagavya + Humic acid) for both kharif and summer season. Application of 100 per cent drip fertigation through water soluble fertilizers along with bio stimulants $\mathrm{T}_{9}$ significantly higher $\mathrm{N}$ content $(4.4$ and $4.1 \%$ at $60 \mathrm{DAT}), \mathrm{P}$ (0.48 and $0.45 \%$ at 60 DAT), K (5.0 and $4.3 \%$ at 60 DAT), Ca (0.43 and $0.42 \%$ at $60 \mathrm{DAT}), \mathrm{Mg}$ (1.5 and $1.5 \%$ at $60 \mathrm{DAT}), \mathrm{Zn}(45.1$ and $44.6 \mathrm{ppm}$ at $60 \mathrm{DAT}) \mathrm{Cu}$ (20.7 and $20.4 \mathrm{ppm}$ at $60 \mathrm{DAT})$ and Fe (260.4 and $259.5 \mathrm{ppm}$ at $60 \mathrm{DAT}$ ) in both kharif and summer seasons.

\section{Introduction}

Chilli (Capsicum annuum L.) is a spice cum vegetable crop belongs to the family Solanaceae. Chillies are nature's wonder. The fruits appear in different sizes, shapes and colours. Chillies have two important qualities, they have biting pungency attributed to capsaicin and captivating red colour due to the pigment capsanthin. Capsaicin is a digestive stimulant, prevents heart diseases and curative for many rheumatic troubles.

Application of water soluble fertilizers through drip irrigation system is gaining importance in present day agriculture to boost the production and productivity of various crops. However, the cost of water soluble fertilizer is very high which restricts the use of fertigation, it is essential to explore the possibility of using normal fertilizer in drip fertigation. Further, Chilli crop requires a balanced fertilizer management for normal growth and development of the crop. It is a heavy feeder of nutrients and high yield can be sustained only through the application of nutrients at optimum doses in balanced proportion. The nutrient requirement also varies with the growth stages of the crop. Among the various factors responsible for higher yield, the use of appropriate quantity of fertilizer at proper time plays a vital role in enhancing the productivity. Hence, planning the nutrient supply to the physiological stages 
of growth development, and considering the soil and climatic characteristics result in high yields with quality produces. Proper scheduling must be planned to provide nutrients at a time when it required by plants. This paves the way to undertake this study by adopting integrated approaches like application of inorganic fertilizers in combination with bio stimulants like liquid biofertilizers, panchagavya and humic acid under drip fertigation on nutrient contents like $\mathrm{N}, \mathrm{P}, \mathrm{K}, \mathrm{Ca}, \mathrm{Mg}, \mathrm{Zn}, \mathrm{Cu}$ and $\mathrm{Fe}$ in chilli.

\section{Materials and Methods}

The experiments were conducted at the College Orchard, Agricultural College and Research Institute, Madurai. The farm is geographically situated at $9^{\circ} 54^{\prime} \mathrm{N}$ latitude and $78^{\circ} 54^{\prime}$ E longitude with an altitude of 147 meters above mean sea level. The cropping period of first crop (kharif), i.e., from June to December 2007 and the second experiment was carried out during summer (January May, 2008). The experiment was laid out in Randomized Block Design with nine treatments replicated thrice with plot size of $45 \mathrm{~m}^{2}$ (10 m x $\left.4.5 \mathrm{~m}\right)$. After uniform leveling, beds were formed at a distance of $1.5 \mathrm{~m}$ and plants were transplanted uniformly with a spacing of $60 / 90 \mathrm{~cm}$ between two pairs 45 $\mathrm{cm}$ between plants were followed.

The recommended dose of 120: 60: $30 \mathrm{~kg}$ NPK ha ${ }^{-1}$ was applied in the experimental plots. Fertigation was scheduled once in three days starting from second week after planting. The fertigation scheduling was planned to meet the crops demand and requirement of the nutrients at different stages of crop growth. Monoammonium phosphate, polyfeed and potassium nitrate of imported grade with water soluble solid fertilizers were used. Urea, Single Super Phosphate and muriate of potash were given as normal fertilizers.
Standard culture operation and necessary crop protection measures were followed during cropping period as per horticulture crop production guide. The data obtained from present investigation were subjected to statistical scrutiny by adopting standard procedure as described by Panse and Sukhatme (1985) and the results were interpreted.

\section{Treatment details}

$\mathrm{T}_{1}=\mathrm{RDF}$ through soil application (120: 60: $30 \mathrm{~kg} \mathrm{NPK} \mathrm{ha}{ }^{-1}$ )

$\mathrm{T}_{2}=75 \%$ RDF as Urea + DAP (as basal) + $\mathrm{KCl}$

$\mathrm{T}_{3}=75 \%$ RDF as Water Soluble Fertilizers

$\mathrm{T}_{4}=100 \% \mathrm{RDF}$ as Urea + DAP (as basal) + $\mathrm{KCl}$

$\mathrm{T}_{5}=100 \%$ RDF Water Soluble Fertilizers

$\mathrm{T}_{6}=\mathrm{T}_{2}+$ liquid biofertilizers + Panchagavya + Humic acid

$\mathrm{T}_{7}=\mathrm{T}_{3}+$ liquid biofertilizers + Panchagavya + Humic acid

$\mathrm{T}_{8}=\mathrm{T}_{4}+$ liquid biofertilizers + Panchagavya + Humic acid

$\mathrm{T}_{9}=\mathrm{T}_{5}+$ liquid biofertilizers + Panchagavya + Humic acid

Note:

Water soluble fertilizers: Poly feed $(19 \% \mathrm{~N}$, $19 \% \mathrm{P}$ and $19 \% \mathrm{~K}) \mathrm{MAP}(12 \% \mathrm{~N}$ and $61 \% \mathrm{P})$ and $\mathrm{KNO}_{3}(13 \% \mathrm{~N}$ and $45 \% \mathrm{~K})$

Liquid biofertilizers (200 $\mathrm{ml}$ /acre), Panchagavya (10 litre/ac) and Humic acid (2 litre/ac) 


\section{Results and Discussion}

The experiments were carried out to find the optimum level of fertigation for higher yield besides quality in chilli. The $\mathrm{N}, \mathrm{P}, \mathrm{K}, \mathrm{Ca}, \mathrm{Mg}$, $\mathrm{Cu}, \mathrm{Zn}$ and $\mathrm{Fe}$ contents in plant were estimated (Tables 1-8). Among the macronutrients, nitrogen encourages vegetative growth and imparts deep green colour to the leaves. In the present investigation, application of 100 per cent water soluble fertilizer along with bio stimulants under fertigation system showed higher nitrogen content in plant.

The presence of some macro and micro nutrients besides total reducing (glucose) sugars in panchagavya. Chemolitho autotrophic nitrifiers (ammonifiers and nitrifiers) in panchagavya increase the ammonia uptake and enhances total $\mathrm{N}$ supply (Papen et al., 2002). This was supported by the studies made by Kanimozhi (2004) in Coleus forskohlii.

Increase in plant nitrogen content due to liquid bio stimulants like Azospirillum inoculation has been observed by several workers in several crops (Christiansen Weniger, 1988; Singleton et al., 2002) (Table 1). In this study also the $\mathrm{N}$ content of chilli was increased due to the inoculation of Azospirillum and the higher content was obtained with liquid formulation. Since plants inoculated with Azospirillum had maximum N content, it is reasonable to think that the inoculation might have enhanced ' $\mathrm{N}$ ' uptake by the plants due to increased availability of $\mathrm{N}$ in the rhizosphere by the activity of the inoculated bacteria. The present result is in agreement with Rao and Rao (1983) wherein they found increased nitrogen content in paddy due to Azospirillum inoculation.

Table.1 Effect of fertigation on $\mathrm{N}$ content (\%) at different crop growth stages of chillies cv.

KKM-1

\begin{tabular}{|c|c|c|c|c|c|c|c|c|}
\hline \multirow{2}{*}{ Treatments } & \multicolumn{5}{|c|}{ Kharif } & \multicolumn{4}{c|}{ Summer } \\
\cline { 2 - 9 } & 60DAT & 90DAT & 120DAT & 180DAT & 60DAT & 90DAT & 120DAT & 180DAT \\
\hline $\mathrm{T}_{1}$ & 3.25 & 3.18 & 3.11 & 2.91 & 3.19 & 3.12 & 3.08 & 2.78 \\
\hline $\mathrm{T}_{2}$ & 3.37 & 3.31 & 3.24 & 3.04 & 3.26 & 3.21 & 3.16 & 2.83 \\
\hline $\mathrm{T}_{3}$ & 3.75 & 3.68 & 3.61 & 3.10 & 3.45 & 3.39 & 3.33 & 2.95 \\
\hline $\mathrm{T}_{4}$ & 3.63 & 3.57 & 3.49 & 2.98 & 3.31 & 3.25 & 3.18 & 2.89 \\
\hline $\mathrm{T}_{5}$ & 3.98 & 3.93 & 3.86 & 3.35 & 3.83 & 3.79 & 3.72 & 3.20 \\
\hline $\mathrm{T}_{6}$ & 3.86 & 3.80 & 3.72 & 3.21 & 3.75 & 3.68 & 3.64 & 3.09 \\
\hline $\mathrm{T}_{7}$ & 4.29 & 4.24 & 4.17 & 3.67 & 4.09 & 4.04 & 3.98 & 3.41 \\
\hline $\mathrm{T}_{8}$ & 4.15 & 4.09 & 4.02 & 3.49 & 3.97 & 3.93 & 3.89 & 3.30 \\
\hline $\mathrm{T}_{9}$ & 4.38 & 4.33 & 4.26 & 3.91 & 4.14 & 4.10 & 4.06 & 3.53 \\
\hline SEd & $\mathbf{0 . 0 8 4}$ & $\mathbf{0 . 0 8 6}$ & $\mathbf{0 . 0 8 6}$ & $\mathbf{0 . 0 7 3}$ & $\mathbf{0 . 0 7 9}$ & $\mathbf{0 . 0 8 1}$ & $\mathbf{0 . 0 8 2}$ & $\mathbf{0 . 0 5 8}$ \\
\hline CD(p=0.05) & $\mathbf{0 . 1 7 9}$ & $\mathbf{0 . 1 8 2}$ & $\mathbf{0 . 1 8 2}$ & $\mathbf{0 . 1 5 5}$ & $\mathbf{0 . 1 6 9}$ & $\mathbf{0 . 1 7}$ & $\mathbf{0 . 1 7 4}$ & $\mathbf{0 . 1 2 3}$ \\
\hline
\end{tabular}


Table.2 Effect of fertigation on P content (\%) at different crop growth stages of chillies cv. KKM-1

\begin{tabular}{|c|c|c|c|c|c|c|c|c|}
\hline \multirow{2}{*}{ Treatments } & \multicolumn{9}{l|}{ Kharif } & \multicolumn{1}{l|}{ Summer } \\
\cline { 2 - 9 } & 60DAT & 90DAT & 120DAT & 180DAT & 60DAT & 90DAT & 120DAT & 180DAT \\
\hline $\mathrm{T}_{1}$ & 0.301 & 0.286 & 0.255 & 0.234 & 0.292 & 0.277 & 0.242 & 0.220 \\
\hline $\mathrm{T}_{2}$ & 0.339 & 0.324 & 0.292 & 0.279 & 0.297 & 0.282 & 0.247 & 0.261 \\
\hline $\mathrm{T}_{3}$ & 0.381 & 0.367 & 0.335 & 0.323 & 0.343 & 0.328 & 0.296 & 0.308 \\
\hline $\mathrm{T}_{4}$ & 0.355 & 0.338 & 0.307 & 0.301 & 0.328 & 0.313 & 0.280 & 0.294 \\
\hline $\mathrm{T}_{5}$ & 0.416 & 0.401 & 0.371 & 0.354 & 0.389 & 0.374 & 0.343 & 0.330 \\
\hline $\mathrm{T}_{6}$ & 0.397 & 0.381 & 0.349 & 0.330 & 0.357 & 0.342 & 0.307 & 0.319 \\
\hline $\mathrm{T}_{7}$ & 0.455 & 0.447 & 0.418 & 0.369 & 0.439 & 0.422 & 0.388 & 0.340 \\
\hline $\mathrm{T}_{8}$ & 0.431 & 0.421 & 0.390 & 0.357 & 0.423 & 0.410 & 0.375 & 0.337 \\
\hline $\mathrm{T}_{9}$ & 0.479 & 0.466 & 0.439 & 0.395 & 0.451 & 0.438 & 0.403 & 0.355 \\
\hline SEd & $\mathbf{0 . 0 1 2 4}$ & $\mathbf{0 . 0 1 2 8}$ & $\mathbf{0 . 0 1 3 0}$ & $\mathbf{0 . 0 1 1}$ & $\mathbf{0 . 0 1 2 9}$ & $\mathbf{0 . 0 1 3 0}$ & $\mathbf{0 . 0 1 3 0}$ & $\mathbf{0 . 0 0 9}$ \\
\hline CD(p=0.05) & $\mathbf{0 . 0 2 6 2}$ & $\mathbf{0 . 0 2 7 0}$ & $\mathbf{0 . 0 2 7 6}$ & $\mathbf{0 . 0 2 3}$ & $\mathbf{0 . 0 2 7 4}$ & $\mathbf{0 . 0 2 7 5}$ & $\mathbf{0 . 0 2 7 5}$ & $\mathbf{0 . 0 2 0}$ \\
\hline
\end{tabular}

Table.3 Effect of fertigation on $\mathrm{K}$ content (\%) at different crop growth stages of chillies cv. KKM-1

\begin{tabular}{|c|c|c|c|c|c|c|c|c|}
\hline \multirow{2}{*}{ Treatments } & \multicolumn{9}{l}{ Kharif } & \multicolumn{2}{l|}{ Summer } \\
\cline { 2 - 9 } & 60DAT & 90DAT & 120DAT & 180DAT & 60DAT & 90DAT & 120DAT & 180DAT \\
\hline $\mathrm{T}_{1}$ & 3.61 & 3.49 & 3.33 & 3.07 & 3.47 & 3.36 & 3.21 & 2.99 \\
\hline $\mathrm{T}_{2}$ & 3.87 & 3.76 & 3.66 & 3.29 & 3.51 & 3.45 & 3.34 & 3.11 \\
\hline $\mathrm{T}_{3}$ & 4.43 & 4.33 & 4.21 & 3.65 & 3.73 & 3.64 & 3.57 & 3.37 \\
\hline $\mathrm{T}_{4}$ & 4.38 & 4.27 & 4.15 & 3.48 & 3.67 & 3.59 & 3.51 & 3.2 \\
\hline $\mathrm{T}_{5}$ & 4.69 & 4.58 & 4.47 & 3.80 & 4.13 & 4.07 & 3.99 & 3.54 \\
\hline $\mathrm{T}_{6}$ & 4.58 & 4.47 & 4.35 & 3.69 & 4.10 & 3.92 & 3.84 & 3.41 \\
\hline $\mathrm{T}_{7}$ & 4.91 & 4.80 & 4.69 & 4.17 & 4.24 & 4.17 & 4.10 & 3.90 \\
\hline $\mathrm{T}_{8}$ & 4.74 & 4.63 & 4.52 & 3.98 & 4.19 & 4.11 & 4.02 & 3.77 \\
\hline $\mathrm{T}_{9}$ & 5.04 & 4.94 & 4.88 & 4.29 & 4.31 & 4.26 & 4.18 & 4.05 \\
\hline SEd & $\mathbf{0 . 1 0 4}$ & $\mathbf{0 . 1 0 2}$ & $\mathbf{0 . 1 0 3}$ & $\mathbf{0 . 0 8 6}$ & $\mathbf{0 . 0 7 1}$ & $\mathbf{0 . 0 7 2}$ & $\mathbf{0 . 0 7 6}$ & $\mathbf{0 . 0 7 9}$ \\
\hline CD(p=0.05) & $\mathbf{0 . 2 1 4}$ & $\mathbf{0 . 2 1 6}$ & $\mathbf{0 . 2 1 9}$ & $\mathbf{0 . 1 8 3}$ & $\mathbf{0 . 1 5 1}$ & $\mathbf{0 . 1 5 3}$ & $\mathbf{0 . 1 6 1}$ & $\mathbf{0 . 1 6 7}$ \\
\hline
\end{tabular}

Table.4 Effect of fertigation on Ca content (\%) at different crop growth stages of chillies cv. KKM-1

\begin{tabular}{|c|c|c|c|c|c|c|c|c|}
\hline Treatments & \multicolumn{9}{l}{ Kharif } & \multicolumn{2}{l|}{ Summer } \\
\cline { 2 - 9 } & 60DAT & 90DAT & 120DAT & 180DAT & 60DAT & 90DAT & 120DAT & 180DAT \\
\hline $\mathrm{T}_{1}$ & 0.253 & 0.234 & 0.213 & 0.199 & 0.229 & 0.210 & 0.188 & 0.178 \\
\hline $\mathrm{T}_{2}$ & 0.265 & 0.251 & 0.230 & 0.201 & 0.250 & 0.233 & 0.214 & 0.194 \\
\hline $\mathrm{T}_{3}$ & 0.298 & 0.283 & 0.264 & 0.229 & 0.287 & 0.268 & 0.249 & 0.213 \\
\hline $\mathrm{T}_{4}$ & 0.278 & 0.265 & 0.243 & 0.221 & 0.274 & 0.255 & 0.236 & 0.200 \\
\hline $\mathrm{T}_{5}$ & 0.387 & 0.376 & 0.357 & 0.322 & 0.353 & 0.336 & 0.318 & 0.282 \\
\hline $\mathrm{T}_{6}$ & 0.363 & 0.349 & 0.330 & 0.295 & 0.339 & 0.320 & 0.301 & 0.265 \\
\hline $\mathrm{T}_{7}$ & 0.419 & 0.408 & 0.389 & 0.361 & 0.398 & 0.379 & 0.362 & 0.329 \\
\hline $\mathrm{T}_{8}$ & 0.411 & 0.399 & 0.380 & 0.345 & 0.402 & 0.384 & 0.366 & 0.331 \\
\hline $\mathrm{T}_{9}$ & 0.431 & 0.419 & 0.402 & 0.373 & 0.418 & 0.401 & 0.383 & 0.353 \\
\hline SEd & $\mathbf{0 . 0 1 5}$ & $\mathbf{0 . 0 1 5}$ & $\mathbf{0 . 0 1 6}$ & $\mathbf{0 . 0 1 5}$ & $\mathbf{0 . 0 1 5}$ & $\mathbf{0 . 0 1 5}$ & $\mathbf{0 . 0 1 5}$ & $\mathbf{0 . 0 1 4}$ \\
\hline CD(p=0.05) & $\mathbf{0 . 0 3 2}$ & $\mathbf{0 . 0 3 3}$ & $\mathbf{0 . 0 3 4}$ & $\mathbf{0 . 0 3 3}$ & $\mathbf{0 . 0 3 2}$ & $\mathbf{0 . 0 3 2}$ & $\mathbf{0 . 0 3 2}$ & $\mathbf{0 . 0 3 0}$ \\
\hline
\end{tabular}


Table.5 Effect of fertigation on $\mathrm{Mg}$ content (\%) at different crop growth stages of chillies cv. KKM-1

\begin{tabular}{|c|c|c|c|c|c|c|c|c|}
\hline \multirow{2}{*}{ Treatments } & \multicolumn{9}{l|}{ Kharif } & \multicolumn{2}{l|}{ Summer } \\
\cline { 2 - 9 } & 60DAT & 90DAT & 120DAT & 180DAT & 60DAT & 90DAT & 120DAT & 180DAT \\
\hline $\mathrm{T}_{1}$ & 1.21 & 1.16 & 1.07 & 0.97 & 1.19 & 1.08 & 0.95 & 0.88 \\
\hline $\mathrm{T}_{2}$ & 1.36 & 1.30 & 1.21 & 1.08 & 1.25 & 1.19 & 1.10 & 0.96 \\
\hline $\mathrm{T}_{3}$ & 1.29 & 1.23 & 1.14 & 1.05 & 1.34 & 1.28 & 1.19 & 1.11 \\
\hline $\mathrm{T}_{4}$ & 1.38 & 1.31 & 1.26 & 1.10 & 1.29 & 1.23 & 1.14 & 1.03 \\
\hline $\mathrm{T}_{5}$ & 1.43 & 1.38 & 1.31 & 1.22 & 1.38 & 1.33 & 1.25 & 1.15 \\
\hline $\mathrm{T}_{6}$ & 1.41 & 1.35 & 1.27 & 1.14 & 1.35 & 1.29 & 1.21 & 1.07 \\
\hline $\mathrm{T}_{7}$ & 1.49 & 1.43 & 1.35 & 1.26 & 1.43 & 1.38 & 1.32 & 1.20 \\
\hline $\mathrm{T}_{8}$ & 1.45 & 1.38 & 1.29 & 1.18 & 1.40 & 1.32 & 1.26 & 1.18 \\
\hline $\mathrm{T}_{9}$ & 1.53 & 1.49 & 1.46 & 1.30 & 1.48 & 1.44 & 1.38 & 1.24 \\
\hline SEd & $\mathbf{0 . 0 2 1}$ & $\mathbf{0 . 0 2 1}$ & $\mathbf{0 . 0 2 3}$ & $\mathbf{0 . 0 2 2}$ & $\mathbf{0 . 0 1 9}$ & $\mathbf{0 . 0 2 3}$ & $\mathbf{0 . 0 2 7}$ & $\mathbf{0 . 0 2 5}$ \\
\hline CD(p=0.05) & $\mathbf{0 . 0 4 5}$ & $\mathbf{0 . 0 4 6}$ & $\mathbf{0 . 0 5 0}$ & $\mathbf{0 . 0 4 8}$ & $\mathbf{0 . 0 4 1}$ & $\mathbf{0 . 0 4 8}$ & $\mathbf{0 . 0 5 8}$ & $\mathbf{0 . 0 5 4}$ \\
\hline
\end{tabular}

Table.6 Effect of fertigation on Zn (ppm) at different crop growth stages of chillies cv. KKM-1

\begin{tabular}{|c|c|c|c|c|c|c|c|c|}
\hline \multirow{2}{*}{ Treatments } & \multicolumn{4}{|c|}{ Kharif } & \multicolumn{4}{c|}{ Summer } \\
\cline { 2 - 9 } & 60DAT & 90DAT & 120DAT & 180DAT & 60DAT & 90DAT & 120DAT & 180DAT \\
\hline $\mathrm{T}_{1}$ & 43.18 & 42.74 & 40.21 & 36.04 & 42.37 & 42.01 & 39.53 & 35.10 \\
\hline $\mathrm{T}_{2}$ & 43.52 & 43.29 & 40.93 & 36.67 & 42.81 & 42.47 & 40.01 & 35.39 \\
\hline $\mathrm{T}_{3}$ & 44.28 & 44.01 & 41.54 & 37.25 & 43.74 & 43.42 & 41.04 & 35.81 \\
\hline $\mathrm{T}_{4}$ & 43.81 & 43.55 & 41.12 & 36.88 & 43.37 & 43.03 & 40.58 & 35.47 \\
\hline $\mathrm{T}_{5}$ & 44.60 & 44.37 & 42.09 & 37.99 & 44.12 & 43.82 & 41.43 & 36.60 \\
\hline $\mathrm{T}_{6}$ & 44.45 & 44.08 & 41.72 & 37.34 & 43.92 & 43.64 & 41.25 & 36.29 \\
\hline $\mathrm{T}_{7}$ & 44.87 & 44.61 & 42.15 & 38.45 & 44.43 & 44.16 & 41.80 & 37.18 \\
\hline $\mathrm{T}_{8}$ & 44.75 & 44.40 & 41.89 & 38.11 & 44.27 & 43.99 & 41.59 & 36.99 \\
\hline $\mathrm{T}_{9}$ & 45.11 & 44.78 & 42.41 & 38.96 & 44.61 & 44.35 & 41.97 & 37.66 \\
\hline SEd & $\mathbf{0 . 1 4 0}$ & $\mathbf{0 . 1 4 4}$ & $\mathbf{0 . 1 5 0}$ & $\mathbf{0 . 2 0 1}$ & $\mathbf{0 . 1 6 3}$ & $\mathbf{0 . 1 7 0}$ & $\mathbf{0 . 1 7 9}$ & $\mathbf{0 . 1 9 3}$ \\
\hline CD(p=0.05) & $\mathbf{0 . 2 9 8}$ & $\mathbf{0 . 3 0 6}$ & $\mathbf{0 . 3 1 9}$ & $\mathbf{0 . 4 2 6}$ & $\mathbf{0 . 3 4 6}$ & $\mathbf{0 . 3 6 1}$ & $\mathbf{0 . 3 7 9}$ & $\mathbf{0 . 4 0 9}$ \\
\hline
\end{tabular}

Table.7 Effect of fertigation on $\mathrm{Cu}(\mathrm{ppm})$ at different crop growth stages of chillies cv. KKM-1

\begin{tabular}{|c|c|c|c|c|c|c|c|c|}
\hline \multirow{2}{*}{ Treatments } & \multicolumn{9}{l|}{ Kharif } & \multicolumn{2}{l|}{ Summer } \\
\cline { 2 - 9 } & 60DAT & 90DAT & 120DAT & 180DAT & 60DAT & 90DAT & 120DAT & 180DAT \\
\hline $\mathrm{T}_{1}$ & 19.47 & 17.98 & 15.19 & 13.77 & 19.33 & 18.02 & 15.23 & 13.59 \\
\hline $\mathrm{T}_{2}$ & 19.61 & 18.14 & 15.38 & 14.09 & 19.47 & 18.12 & 15.31 & 13.71 \\
\hline $\mathrm{T}_{3}$ & 19.93 & 18.47 & 15.76 & 14.15 & 19.61 & 18.26 & 15.43 & 14.01 \\
\hline $\mathrm{T}_{4}$ & 19.75 & 18.30 & 15.57 & 13.98 & 19.53 & 18.17 & 15.35 & 13.87 \\
\hline $\mathrm{T}_{5}$ & 20.01 & 18.65 & 16.11 & 14.35 & 19.89 & 18.45 & 15.68 & 14.19 \\
\hline $\mathrm{T}_{6}$ & 19.97 & 18.58 & 15.93 & 14.21 & 19.81 & 18.39 & 15.59 & 13.98 \\
\hline $\mathrm{T}_{7}$ & 20.48 & 19.13 & 16.57 & 15.04 & 20.06 & 18.57 & 15.81 & 14.49 \\
\hline $\mathrm{T}_{8}$ & 20.29 & 18.95 & 16.31 & 14.93 & 19.97 & 18.49 & 15.73 & 14.27 \\
\hline $\mathrm{T}_{9}$ & 20.67 & 19.36 & 16.77 & 15.19 & 20.35 & 18.81 & 15.96 & 14.63 \\
\hline SEd & $\mathbf{0 . 0 8 5}$ & $\mathbf{0 . 0 9 9}$ & $\mathbf{0 . 1 1 5}$ & $\mathbf{0 . 1 1 0}$ & $\mathbf{0 . 0 7 0}$ & $\mathbf{0 . 0 5 3}$ & $\mathbf{0 . 0 5 3}$ & $\mathbf{0 . 0 7 4}$ \\
\hline CD(p=0.05) & $\mathbf{0 . 1 8 1}$ & $\mathbf{0 . 2 1 0}$ & $\mathbf{0 . 2 4 5}$ & $\mathbf{0 . 2 3 3}$ & $\mathbf{0 . 1 4 8}$ & $\mathbf{0 . 1 1 3}$ & $\mathbf{0 . 1 1 4}$ & $\mathbf{0 . 1 5 8}$ \\
\hline
\end{tabular}


Table.8 Effect of fertigation on Fe (ppm) at different crop growth stages of chillies cv. KKM-1

\begin{tabular}{|c|c|c|c|c|c|c|c|c|}
\hline \multirow{2}{*}{ Treatments } & \multicolumn{1}{l}{ Kharif } & \multicolumn{1}{l|}{ Summer } \\
\cline { 2 - 9 } & 60DAT & 90DAT & 120DAT & 180DAT & 60DAT & 90DAT & 120DAT & 180DAT \\
\hline $\mathrm{T}_{1}$ & 247.29 & 240.52 & 231.79 & 211.04 & 245.81 & 238.79 & 229.66 & 208.27 \\
\hline $\mathrm{T}_{2}$ & 249.97 & 243.18 & 234.42 & 216.35 & 247.43 & 240.52 & 231.53 & 212.30 \\
\hline $\mathrm{T}_{3}$ & 254.41 & 248.55 & 239.70 & 225.17 & 251.58 & 244.75 & 235.82 & 219.73 \\
\hline $\mathrm{T}_{4}$ & 251.81 & 244.15 & 236.63 & 220.03 & 249.47 & 242.62 & 233.78 & 215.18 \\
\hline $\mathrm{T}_{5}$ & 257.98 & 251.07 & 245.19 & 226.11 & 255.25 & 249.21 & 240.50 & 222.47 \\
\hline $\mathrm{T}_{6}$ & 256.67 & 249.79 & 242.85 & 223.47 & 254.97 & 248.38 & 239.61 & 219.61 \\
\hline $\mathrm{T}_{7}$ & 258.34 & 253.51 & 247.37 & 231.19 & 257.69 & 251.87 & 243.17 & 227.34 \\
\hline $\mathrm{T}_{8}$ & 257.64 & 251.94 & 245.94 & 229.07 & 255.81 & 250.64 & 241.95 & 224.68 \\
\hline $\mathrm{T}_{9}$ & 260.41 & 255.55 & 249.07 & 234.32 & 259.47 & 254.39 & 246.83 & 230.15 \\
\hline $\mathrm{SEd}$ & $\mathbf{0 . 9 4 8}$ & $\mathbf{1 . 0 9 4}$ & $\mathbf{1 . 3 1 9}$ & $\mathbf{1 . 5 8 7}$ & $\mathbf{1 . 0 1 8}$ & $\mathbf{1 . 1 6 2}$ & $\mathbf{1 . 2 3 8}$ & $\mathbf{1 . 5 3 7}$ \\
\hline $\mathbf{C D}(\mathbf{p}=\mathbf{0 . 0 5})$ & $\mathbf{2 . 0 1 1}$ & $\mathbf{2 . 3 2 0}$ & $\mathbf{2 . 7 9 6}$ & $\mathbf{3 . 3 6 6}$ & $\mathbf{2 . 1 5 9}$ & $\mathbf{2 . 4 6 4}$ & $\mathbf{2 . 6 2 6}$ & $\mathbf{3 . 2 5 9}$ \\
\hline
\end{tabular}

Phosphorus plays key role in the plants energy transfer system (Table 2). The present study, relatively maximum phosphorus content in plant was noticed with application of 100 per cent water soluble fertilizer along with bio stimulants. This finding is in accordance with Papadopoulos (1992) in potato cv. Spunta.

Potassium, being a protoplasmic factor is an essential plant nutrient. Many enzymes are activated by potassium and potassium is also involved in photo and oxidative phosphorylation thus augmenting the energy required for fruit growth. Drip fertigation with 100 per cent water soluble fertilizers along with bio stimulants enhanced the absorption of potassium in both the seasons of crop growth. Fontes et al., (2000) opined that application of $\mathrm{N}$ and $\mathrm{K}$ in combination with drip irrigation increased the yield by the way of maximizing the mobility of the nutrients around the root zone (Table 3). These results are in agreement with those obtained by ElGizawy (1993) and Kavitha (2005) in tomato. The potassium content in the chilli plant exerted a similar pattern as that in the case of nitrogen and phosphorus. The transformation reactions that took place led to greater availability of potassium in the soil and consequently resulted in the better utilization by the plant. It is also possible that the fertigation combined with water soluble fertilizer might have activated the physiological processes for the rapid absorption and utilization of the nutrient for the primary metabolic processes. Similar findings were reported by Ghanta and Mitra (1993) and Singh et al., (1995).

\section{References}

Christiansen-Weniger C 1988 An influence of plant growth substances on growth and nitrogenase activity from Azospirillum brasilense. In: Azospirillum. IV. Genetics, physiology and ecology. Klingmüller W, Springer-Verlag, Berlin, Heidelberg. p.141-149.

El-Gizawy AM, Abdallah MMF, Gomaa HM and Mohamed SS 1993 Effect of different shading levels on tomato plants. 2. Yield and fruit quality. Acta. Hort. 323: 349-354.

Fontes PCR, Sampaio RA and FingerFL2000 Fruit size, mineral composition and quality of trickle irrigated tomatoes as affected by potassium rates. Pesquisa Agropecuaria Brasileira 35(1): 21-25.

Ghanta PK and Mitra SK 1993 Effect of 
micronutrients on growth, flowering, leaf nutrient content and yield of banana cv. Giant Governor. Crop Res. 6(2): 284287.

Kanimozhi C 2004 Standardization of organic production packages for Coleus forskohlii Briq. M.Sc. (Horti.) thesis, Tamil Nadu Agricultural University, Coimbatore.

Kavitha M 2005 Studies on effect of shade and fertigation on growth and yield of tomato (Lycopersicon esculentum Mill.) Hybrid Ruchi. Ph.D. (Hort.) thesis, Tamil Nadu Agricultural University, Coimbatore.

Panse VG and Sukhatme PV 1985 Statistical methods for agricultural workers. ICAR, New Delhi. p. 134-192.

Papadopoulos I 1992 Phosphorus fertigation of trickle irrigated potato. Fert. Res. 31(1): 9-13.

Papen H, GeblerA,Zumbusch E and RennenhegH $2002 \quad$ Chemolitho autotrophic nitrifiers in the phyllosphere of a spruce ecosystem receiving high nitrogen input. Current Microbiol. 44: 56-60.

Rao JLN and RaoVR 1983 Nitrogenase activity in the rice rhizosphere soil as affected by Azospirillum inoculation and fertilizer nitrogen under upland condition. Curr. Sci. 52: 686-688.

Singh AK, Singh K, Singh UN, Raju MS and SinghJP 1995 Effect of potassium, zinc and iron on yield, post-harvest and nutrient uptake in French bean (Phaseolus vulgaris L.). J. Karnataka Res. 11(1): 75-80.

Singleton PW, Keyser $\mathrm{HH}$ and Sande ES 2002 Development and evaluation of liquid inoculants. In: Inoculants and nitrogen fixation of legumes in Vietnam. (eds., D. Herridge) ACIAR Proceedings, 109e, 52-66.

\section{How to cite this article:}

Ciba, C. and Syamala, M. 2017. Drip Fertigation's Effect on Nutrient Contents of Chiili (Capsicum annuum). Int.J.Curr.Microbiol.App.Sci. 6(11): 3314-3320. doi: https://doi.org/10.20546/ijcmas.2017.611.388 